\title{
Kämpfen für die Sache Allahs: Zur Genese dschihadistischer Identitäten
}

\author{
Amir SHEIKHZADEGAN ${ }^{1}$
}

Universität Freiburg i. Ü.

\begin{abstract}
Obwohl die Entstehung des Dschihadismus auf das Zusammenspiel diverser Faktoren auf verschiedenen sozietalen Ebenen zurückzuführen ist, liegen die Gründe, weshalb sich ein Individuum dem Dschihadismus anschliesst, am ehesten in jenen gesellschaftlichen Dynamiken, die bei diesem neue Selbst- und Weltbilder entstehen lassen. Deshalb setzt ein adäquates Verständnis der Entstehungsgründe des Dschihadismus auch Kenntnisse über die Genese dschihadistischer Identitäten voraus. Diese Einsicht hat in den letzten Jahren ein beachtliches Repertoire an einschlägigen Studien hervorgebracht, das durch die Vielfalt der Fragestellungen sowie eine grosse Varianz an theoretischen und methodologischen Zugängen gekennzeichnet ist.

Trotz dieser beachtlichen Menge und Vielfalt weist der Forschungsstand zurzeit einen disparaten und fragmentierten Zustand auf, weshalb die gewonnenen Erkenntnisse nur unzureichend zu einer Vertiefung unseres Verständnisses dieses Phänomens beitragen. Eine solche Konvergenz bedarf als erstes einer Systematisierung der identitätsrelevanten Ansätze und Studien. Der vorliegende Beitrag nimmt sich der letzteren Herausforderung an, in dem er anhand einer Literatur-Review exemplarische Ansätze und Studien bezüglich der dschihadistischen Identitäten unter der Berücksichtigung verschiedener Facetten der Identität systematisch untersucht. Dabei wird zum einen zwischen den sozietalen Mikro-, Meso- und MakroEbenen unterschieden. Zum anderen wird versucht, auf jeder dieser Ebenen verschiedene Facetten der Identität aufzuzeigen.
\end{abstract}

Keywords: Islam, Dschihad, Dschihadismus, Identität, kollektive Identität

\section{Einführung}

Blättert man durch die westlichen Medien der 1980er Jahre, so ist man vermutlich überrascht, zu erfahren, dass die afghanischen Mujahidin (wörtlich: die Dschihad-Ausübenden) damals

\footnotetext{
${ }^{1}$ Amir Sheikhzadegan, Dr., ist assoziierter Sozialforscher im Bereich Soziologie, Sozialpolitik und Sozialarbeit und am Schweizerischen Zentrum für Islam und Gesellschaft (SZIG) der Universität Freiburg i. Ü. Seine Forschungsbereiche umfassen politischen Islam, spirituelle Transformation, muslimische Seelsorge und Identität (asheikhzadegan@yahoo.com).
} 
als Helden eines Befreiungskampfes gegen die sowjetische Besatzungsmacht zelebriert wurden. Ein Jahrzehnt später erweckte der Dschihad, der nun in Europa angekommen war - dies als Folge der Verfolgung der Islamisten in Algerien ab $1992^{2}$ - kaum mehr Sympathien.

Genährt wurden dschihadistische Bewegungen durch starke Vernetzung der Weltgesellschaft im Prozess der sogenannten Globalisierung. So konnten die Rufe der Dschihadist*innen nach der Solidarität der globalen Umma nicht zuletzt dank der Erfindung der neuen Medien (Internet, Satellitenfernsehen, Smartphone, etc.) global wahrgenommen werden.

Obschon der Dschihadismus geradezu durch Prozesse der Globalisierung eine globale Ausstrahlung erlangt hat, sehen deren Ideologen ihre Mission darin, die durch Globalisierung erleichterte Diffusion westlicher Verhaltensmuster zu verhindern, die Machtstrukturen der Weltgesellschaft, die sie für die Unterdrückung der muslimischen Völker verantwortlich machen, zu bekämpfen und eine neue, auf islamische Prinzipien beruhende Weltordnung zu schaffen.

Weshalb aber vermag der Dschihadismus, der eine scheinbar antimodernistische Ideologie verheisst, gerade junge Menschen in modernen Kontexten zu faszinieren, ihre Selbst- und Weltbilder zu beeinflussen, und sie zu terroristischen Handlungen in ihren Heimatländern oder im Ausland zu animieren?

Diese brisante Frage hat zahlreiche Wissenschaftler*innen in einer Vielzahl von Disziplinen dazu bewegt, sich mit den identitären Aspekten des Dschihadismus zu befassen. Dennoch weist der diesbezügliche Forschungsstand eher einen fragmentierten und disparaten Charakter auf, so dass die bisherigen Erkenntnisse nur unzureichend zu einem vertieften Verständnis dieses Sachverhalts beitragen. Dies rührt u.a. daher, dass ein interdisziplinärer Austausch selten stattfindet.

Eine solche Konvergenz bedarf als Erstes einer Systematisierung der identitätsrelevanten Ansätze und Studien. Der vorliegende Beitrag nimmt sich der letzteren Herausforderung an, in dem er Studien sowie theoretische Ansätze bezüglich dieses Zusammenhangs, unter der Berücksichtigung verschiedener Facetten der Identität, systematisch bespricht. Bei der Auswahl der Studien wird darauf geachtet, dass verschiedene Facetten der Identität auf der Mikro-, Meso- und Makroebene integriert werden. ${ }^{3}$ In diesem Sinne haben die besprochenen Studien einen eher exemplarischen Charakter und sollten dazu dienen, die Vielschichtigkeit und Varianz der Identität im Kontext des Dschihadismus aufzuzeigen.

Der Beitrag verfolgt zweierlei Ziele: Zum einen liefert er eine Übersicht über Studien und Ansätze, die Kenntnisse über die Genese und Merkmale dschihadistischer Identitäten vermitteln. Zum anderen gliedert er die besprochenen Beiträge nach der Ebene/Facette der Identität, die sie bearbeiten.

\footnotetext{
${ }^{2}$ Die von der militanten algerischen Organisation Groupe Islamique Armé (GIA) verübten Gewalttaten in Europa und in Algerien in den 1990er Jahren lösten in der europäischen Öffentlichkeit grosse Betroffenheit aus und führten zu zahlreichen negativ konnotierten Kommentaren und Berichten in den Medien (siehe etwa Hehn 1996; Lueders 1997; Meiler 1998; oder den Le Monde Artikel von 26.12.1996 betitelt mit La France réaffirme qu'elle ne cédera pas au chantage des terroristes islamistes).

${ }^{3}$ Ein erster Schritt in Richtung einer Multiebenen-Analyse ist bereits von Rahimullah/Larmar/Abdalla (2013) in Bezug auf die Psychologie des Dschihadismus beschritten worden.
} 
Der Beitrag geht als Nächstes auf begriffliche Klarstellungen und Differenzierungen ein. Dann werden theoretische Ansätze wie auch empirische Befunde auf der Makro-, Meso- und Mikroebene besprochen. Anschliessend werden Ansätze und Studien herangezogen, die sich einer multidimensionalen Perspektive bedienen. Im letzten Abschnitt wird der Beitrag zusammenfassend abgerundet und mit einem Ausblick beendet.

\section{Zu den Begriffen}

In diesem Abschnitt wird zunächst der Dschihadismus in Abgrenzung zum klassischen Dschihad definiert und dessen Vielschichtigkeit aufgezeigt. In einem zweiten Schritt wird dann der Begriff der Identität unter Berücksichtigung ihrer verschiedenen Dimensionen diskutiert.

\section{Dschihadismus}

Zunächst einmal soll eine Unterscheidung zwischen dem klassischen Dschihad und dem gegenwärtigen Dschihadismus vorausgeschickt werden (Ashour 2015: 174). Klassischer Dschihad - in dessen kriegerischen Variante ${ }^{4}$ - musste jeweils von einer zentralen Stelle (einer Instanz mit höchster staatlicher und religiöser - oder zumindest religiöser - Autorität) angekündigt werden: von einer zentralen Stelle, weil es sonst ein Chaos entstehen würde; und von höchster religiöser Stelle, weil die Ausrufung des Dschihad wie jedes andere religiöse Gutachten ein solides theologisches Wissen voraussetzt. ${ }^{5}$ Die letztere Bedingung war insofern wichtig, als im Islam die Wahrung von Leib und Gut, sowohl des eigenen als auch der anderen, als die Pflicht jedes Gläubigen betrachtet wird. Aus dieser Überlegung heraus müsste sich die zentrale islamische Autorität nach einer sorgfältigen Berücksichtigung aller theologischen Erwägungen für oder gegen eine Ankündigung des Dschihad aussprechen. Ferner lässt sich im klassischen Dschihad, zumindest theoretisch, zwischen defensivem Dschihad (Dschihad zur Verteidigung von muslimischen Ländern vor Aggressoren) und offensivem Dschihad (Eroberungskriege zur Ausbreitung des Islams) unterscheiden. Während der offensive Dschihad in der Regel von einem rechtmässigen Kalifen angekündigt wurde, hat der defensive Dschihad immer als legitim gegolten (Wiktorowicz 2005: 83).

Was den Dschihadismus anbelangt, muss man Sedgwick (2015: 35-39) zufolge zwischen dem wide understanding of jihadism, der am ehesten im Journalismus vorzufinden ist, und einem narrow understanding dieses Begriffs, der in der Geschichtswissenschaft üblich ist, un-

\footnotetext{
${ }^{4}$ Das arabische Wort Dschihad (vom Stamm dsch-h-d) bedeutet Anstrengung und wird in der Lehre des Islams als Anstrengung auf dem Pfad Allahs (dschihad fi sabil al-llah) verwendet. Der grosse islamische Gelehrte Abu Hamed Mohammad Ghazzali (1058-1111) hat den Dschihad in zwei Typen untergegliedert: grosser Dschihad im Sinne der Anstrengung gegen nafs (Triebseele) und kleiner Dschihad im Sinne des Krieges (siehe Sherif 1975: 45).

${ }^{5}$ Selbst der klassische Dschihad war kein monolithisches Phänomen, sondern variierte zwischen doktrinärer Orthodoxie und Pragmatismus, ja sogar Opportunismus. Diesbezüglich schlägt Heck (2004) die folgende Differenzierung vor: "The early and classical literature on jihad examined here can be broadly divided into two categories: one which understands jihad to serve Islamic hegemony and another which understands it to serve Islamic society. The former-largely an imperial construct-is framed in political and territorial terms, the latter in religious and communal terms" (Heck 2004: 95).
} 
terscheiden. Was das engere Verständnis anbelangt, lässt sich wiederum zwischen Dschihadismus im Sinne der bewaffneten Aufstände in der vormodernen islamischen Welt (in der Regel gegen die Kolonialmächte) und dem modernen Dschihadismus in der Gegenwart unterschieden. Der Letztere, der im Fokus dieses Beitrages steht, wird von Ashour (2015: 174) folgendermassen definiert: "Jihadism is a modern Islamist ideology which believes that armed confrontation with political rivals is a theologically legitimate and instrumentally efficient method for socio-political change".

Um ihre Kampfhandlungen theologisch zu legitimieren, durchsuchen die Dschihadist*innen die vorhandene Quellen, vom Quran bis zu den Schriften der zeitgenössischen salafistischen ${ }^{6}$ Gelehrten. Damit findet eher eine Anpassung der theologischen Argumentation an die Ziele der Dschihadist*innen statt als eine ernsthafte Ausrichtung an den theologischen Grundlagen. ${ }^{7}$ Ganz davon zu schweigen, dass der religiöse Rahmen für manche Dschihadist*innen primär dazu dient, ihre Abenteuerlust, Gewaltaffinität oder Rachegelüste auszuleben (siehe etwa Frindte/Slama/Dietrich/Pisoiu/Uhlmann/Kausch 2016: 12; Hafez 2009: 80; Moghadam 2008a: 256. Für eine Analyse der Motive der terroristischen Handlungen allgemein siehe Cottee/Hayward 2011 ${ }^{8}$ ).

Welche Charakteristika eine dschihadistische Bewegung besitzt, hängt davon ab, wie sehr sich die jeweiligen Ideologen der Theologie verpflichtet fühlen, wie fundiert ihr theologisches Wissen ist, welche theologische Positionen sie vertreten, auf welchen Kontext sie sich beziehen und welche Ziele sie befolgen. Dies erklärt die grosse Varianz dschihadistischer Bewegungen, die in den letzten Jahrzehnten in Erscheinung getreten sind. ${ }^{9}$

Wie Moghadam (2008b) aufgezeigt hat, handelt es sich bei den Dschihadismen aus folgenden Gründen um Ideologien: Sie sind jeweils in einem spezifischen Kontext verankert, sprechen eine spezifische Ingroup auf deren grievances (Leidensgeschichten, Missstände) ${ }^{10}$ an, führen diese Missstände auf die Einwirkungen der Feinde des Islams und die daraus resultierende Entfremdung der Ingroup vom echten Islam zurück, konstruieren eine kollektive Identität im Sinne des wir versus andere und stellen den Dschihad als die heilbringende Lösung dar.

Vor dem Hintergrund dieser Vielfalt stellt sich die Frage, ob der Analyserahmen des vorliegenden Beitrags nicht zu weit gesteckt ist. Während diese Frage aus der Sicht eines Historikers möglicherweise bejaht werden könnte, macht es aus der soziologischen Warte Sinn, in

\footnotetext{
${ }^{6}$ Salafismus ist die Bezeichnung für jene Strömungen innerhalb des sunnitischen Islams, die sich unter der Ablehnung jeglicher historischer Entwicklungen des Islams an die sogenannten al-salaf al-salih (die frommen Altvorderen) orientieren. Die Frage, wie das Islam-Verständnis der „frommen Altvorderen“ auszulegen sei, hat aber zu grossen Meinungsverschiedenheiten unter den salafistischen Gelehrten geführt (siehe hierzu etwa die Beiträge des 2014 von Behnam und Fouad herausgegebenen Sammelbandes Salafismus: Auf der Suche nach dem wahren Islam).

${ }^{7}$ Wie Slavicek (2008) aufgezeigt hat, ziehen Dschihadist ${ }^{*}$ innen zur Rechtfertigung ihrer Praktiken und Glaubenssätze Argumente orthodoxer Gelehrten aus allen Epochen der islamischen Geschichte heran.

${ }^{8}$ In ihrer Metaanalyse des Terrorismus schlussfolgern Cottee und Hayward (2011), dass Teilnahme an terroristischen Handlungen auf existentielle Motive zurückzuführen ist, wobei drei Motive, nämlich der Wunsch nach Aufregung (the desire for excitement), der Wunsch nach einem ultimativen Lebenszweck (the desire for ultimate meaning) und der Wunsch nach ehrenhafter Ruhm (the desire for glory) hervorstechen.

${ }^{9}$ Für Typologien der dschihadistischen Bewegungen siehe etwa González Mendelejis (2019); Hegghammer (2010); Hemmingsen (2010: 233); Robinson (2017) und Wagemakers (2017).

${ }^{10}$ Für eine detaillierte Analyse der Leidensgeschichten der Dschihadist*innen siehe Stern (2003).
} 
dieser bunten Landschaft nach allgemeinen Merkmalen dschihadistischer Identitäten und den Umständen ihrer Entstehung zu suchen, denn allen diesen Bewegungen gemeinsam sind religiös begründete Ideologien, die den Einsatz von Gewalt durch den Kampffür die Sache Allahs rechtfertigen.

\section{Identität}

Identität ist ein schillernder Begriff (Brewer 2001: 115; Straub 2000: 167; Schmidhuber 2010: 8), mit dem sich Human- und Sozialwissenschaften aus unterschiedlichen Perspektiven und Erkenntnisinteressen seit geraumer Zeit beschäftigen. Einfach ausgedrückt, bringt dieser Begriff das Selbstverständnis der Menschen zum Ausdruck: „Die klassische Identitätsfrage ,Wer bin ich?' wird erst möglich durch die spezifische Fähigkeit des Menschen zur Selbstreflexivität, seiner Möglichkeit, sich selbst zugleich Subjekt und Objekt zu sein und in ein Verhältnis zu sich selbst treten zu können" (Lucius-Hoene/Deppermann 2004: 47).

Neben den auf das Individuum bezogenen, identitären Konstruktionsleistungen ergeben sich auch Identifikationen auf der Mesoebene, die durch den Bezug eines Individuums zu sozialen Gruppen, Milieus, Szenen, Netzwerken oder Organisationen zustande kommen. Dieser Typus der Identifikation verleiht dem Individuum Gefühle der Zugehörigkeit sowie sozialen Halt und Orientierung.

Jenseits dieser beiden Ebenen entstehen in Gemeinschaften auch Narrative, welche die Zugehörigkeit der Individuen zu jeweiligen Kollektiven zum Ausdruck bringen sollen. Venema (2000) bringt dieses Gedankengut folgendermassen auf den Punkt: "By organizing historical events into a narrative unity, communities and individuals can offer testimony of who they are and how they wish to mark their existence in the world" (Venema 2000: 93; siehe auch Lucius-Hoene/Deppermann 2004: 49; Van Stekelenburg 2013).

Trotz der Vielfalt der Annäherungen an diesen Begriff gibt es unter den zeitgenössischen Identitätstheoretikern weitgehende Übereinstimmung darüber, dass Identität eine vielschichtige und dynamische Entität ist, die von Individuen und sozialen Gruppen in einem dialektischen und interaktiven Prozess kontinuierlich konstruiert wird (Brewer 2001; Keupp/Ahbe/Gmür/Höfer/Mitzscherlich/Kraus/Straus 2008; Lucius-Hoene/Deppermann 2004; Sen 2006; Straub 2018).

Da vielen Dschihadismus-Studien nur ansatzweise identitätsrelevante Kenntnisse entnommen werden können und weil die seltenen, identitätstheoretisch fundierten Studien auch noch auf unterschiedlichen Theorien basieren, kann die Diskussion in diesem Beitrag nicht nach den Inhalten einer einzigen, sich bewährten Identitätstheorie strukturiert werden. Stattdessen wird auf jene Aspekte der Identität fokussiert, deren Relevanz für den Dschihadismus in der Literatur gut dokumentiert ist. Dabei wird zwischen identitären Konstruktionen auf der Makro-, Meso- und Mikroebene unterschieden. Was die identitären Facetten anbelangt, werden kollektive Identitäten, dualistische Weltanschauung und Zugehörigkeit zur globalen Umma auf der Makroebene, identitäre Beeinflussung und Gruppenzugehörigkeit auf der Mesoebene und Adoleszenz, (Re-)Konversion sowie Agency auf der Mikroebene untersucht.

Kurzum, wie die obigen Definitionen gezeigt haben, handelt es sich bei beiden Begriffen Identität und Dschihadismus um komplexe Begriffe. Während Identität sich durch Viel- 
schichtigkeit und Multidimensionalität auszeichnet, lässt sich Dschihadismus als ein variantenreiches Phänomen charakterisieren.

\section{Relevanz der Identität auf der Makroebene}

In diesem Abschnitt wird die Relevanz der Identität für Dschihadismus auf der Makroebene anhand dreier Dimensionen besprochen: kollektive Identität, dualistische Weltanschauung und die Zugehörigkeit zur globalen Umma.

\section{Kollektive Identitäten}

Dem vorwiegend in den 1990er Jahren entfachten Dschihadismus ist eine Ideologisierung des Islams vorausgegangen ist, die in den 1920er Jahren einsetzte und mit zunehmendem Legitimitätsverlust modernistischer Regimes in weiten Teilen der islamischen Welt zum islamischen Wiedererwachen führte. Dieses neue Bewusstsein sollte bald zu politischen Umwälzungen in einigen islamischen Ländern führen. In Ägypten fand die Rückbesinnung zum Islam in den Schriften Sayyid Qutbs ihren radikalsten Ausdruck. In Iran wurde 1979 das pro-westliche, modernistische Monarchie-Regime durch eine islamisch artikulierte Revolution gestürzt. Selbst das eher traditionsbewusste, dennoch pro-westliche, Saudi-Arabien erlebte 1979 einen anti-modernistisch/anti-westlichen Aufstand einer salafistischen Gruppierung. In den Folgejahren kam es zu ähnlichen Umbrüchen in einigen anderen Ländern, etwa in Algerien durch den Front Islamique du Salut (FIS) oder in Sudan durch die National Islamic Front (NIF).

Dass diesem islamischen Wiedererwachen dschihadistische Bewegungen folgten, ist allen voran den Invasionen der Sowjet-Union und der USA in islamische Länder, aber auch der Unterdrückung der islamistischen Bewegungen, etwa in Algerien, Ägypten, Tschetschenien oder in den von Israel besetzten palästinensischen Gebieten, geschuldet (siehe Esposito 2002; Hegghammer 2011; Kepel 2006, 2011; Stern/Berger 2015).

Somit sind die im Entstehen begriffenen kollektiven Identitäten, die aus dem sogenannten islamischen Wiedererwachen in verschiedenen islamisch geprägten Gesellschaften hervorgingen, teilweise durch eine Vielzahl kollektiver Identitäten der dschihadistischen Bewegungen überschattet, wenn nicht gar verdrängt worden. Bei diesen neuen, kollektiven Selbst- und Weltbildern sind die identitären Abgrenzungen nicht nur gegen imperialistische Mächte und deren lokalen Verbündeten ausgerichtet, sondern auch gegen alle Musliminnen, die das Islam-Verständnis der Dschihadist ${ }^{*}$ innen nicht teilen.

\section{Dualistische Weltanschauung}

Die Dichotomie der Hizb-allah (der Partei Gottes) versus der Hizb-ash-shaytan (der Partei Satans) hat in der islamischen Orthodoxie eine lange Tradition und wurde, u.a., vom Gelehrten Taqi Ibn Taymiyya (1263-1328), der für viele Salafist*innen als Inspirationsquelle gilt, aufgegriffen und diskutiert. Dabei ging es den orthodoxen Gelehrten darum, eine scharfe Linie zwischen den wahren Gläubigen und den sogenannten Ungläubigen/Häretikern/Apostaten zu ziehen. Während man die ersteren der Partei Gottes zuschrieb, wurden die letzteren der Gefolgschaft Satans bezichtigt. Diese dichotome Weltanschauung 
wurde vom radikalen ägyptischen Islamisten Sayyid Qutb (1906-1966) wieder aufgenommen und elaboriert. Sein Schüler Muhammad Qutb sowie sein Anhänger Abdullah Azzam übertrugen wiederum diese radikale Weltanschauung an die zwei Führer der al-Qaida, Osama Bin Laden (1957-2011) und Aiman az-Zawahiri (*1951-). Somit fand dieser Dualismus Eingang in dschihadistische Bewegungen und lieferte diesen zugleich eine theologische Rechtfertigung für ihre Gewalttaten. Wiktorowicz (2005: 81) schreibt dazu:

Zawahiri adopted both Qutb's Manichean view of the world and his unwavering desire to establish an Islamic state at any cost, using violence if necessary. This dichotomous struggle for God's sovereignty on earth eliminates the middle ground and sets the stage for a millennial, eschatological battle between good and evil. (Wiktorowicz 2005: 81)

Der Rückgriff auf diese Dichotomie ist aber keineswegs auf sunnitische Islamisten beschränkt. Der Führer der iranischen Revolution von 1979, der schiitische Gelehrte Ayatollah Ruhollah Khomeini (1902-1989), bezeichnete etwa zu Beginn der 1980er Jahre seine Anhänger als die Gefolgschaft Gottes, während er den iranischen König wie auch die USA dämonisierte (Sheikhzadegan 2003: 222).

Die Ideologie des globalen Dschihadismus baut auf dem Narrativ des Kampfes des Islams gegen den Westen und die USA, bzw. des Kampfes des Islams gegen die „Verschwörung der Kreuzzüger und Juden“ (Hegghammer 2011: 299) auf. Dabei ist nicht zu übersehen, dass diese Weltdeutung durch ähnliche Gegenerzählungen seitens führender westlicher Akteure verstärkt wird. Es sei in diesem Zusammenhang daran erinnert, dass US-Präsident George Bush Junior (2001-2009) am 16.09.2001 einen Kreuzzug gegen den Terrorismus und am 29.01.2002 einen Kampf gegen die axis of evil (Achse des Bösen) beschworen hat (siehe auch Maddox 2003).

Aus der Warte der dualistischen Weltanschauung weist auch Agnews (2010) StrainTheorie des Terrorismus ein hohes Erklärungspotential für Dschihadismus auf. Gemäss dieser Theorie ist Terrorismus dann am wahrscheinlichsten, wenn Menschen ein kollektives Leiden teilen, das massiv ist, das mit zivilen Opfern verbunden ist, das als ungerecht empfunden wird, das von übermächtigen Anderen auferlegt wird, die auch noch von ihren Zivilisten unterstützt werden, zu denen die Opfer keine oder nur lose Kontakte haben. Mit anderen Worten, es entsteht unter den erwähnten Bedingungen eine dichotome Weltanschauung von uns, als dem Kollektiv der Unterdrückten, versus den „Anderen“ als dem Kollektiv der Unterdrücker, die $\mathrm{zu}$ terroristischen Handlungen animiert und diese legitimiert. ${ }^{11}$

Von zentraler Bedeutung in diesem Ansatz ist, dass das Kollektiv der Unterdrückten den übermächtigen Anderen nicht auf Augenhöhe, etwa durch eine reguläre Armee, begegnen kann, und deshalb auf Terrorismus - als die Waffe des Schwächeren - zurückgreift. ${ }^{12}$ Des

\footnotetext{
${ }^{11}$ Um hierzu ein Beispiel zu erwähnen, zeigten Schuurman, Bakker und Eijkman (2018) in ihrer Studie über eine holländische Dschihadisten-Gruppe auf, dass bei den strukturellen Vorbedingungen der Entstehung dieser Gruppierung die geopolitical grievances hervorstachen.

${ }^{12}$ In einem Interview mit dem Blatt The Irish Times (Marlowe 2014: 5) brachte der israelische Journalist Gideon Levy diesen Punkt im Kontext des Israel-Palästina-Konflikts wie folgt auf den Punkt: "Any Palestinian terrorist would rather sit in
} 
Weiteren macht die Bedingung, dass die übermächtigen Anderen von ihren Zivilisten unterstützt werden, zu denen die Angehörigen des schwächeren Kollektivs keine oder nur lose Kontakte haben, die Gefährdung der Zivilisten bei den terroristischen Handlungen des schwächeren Kollektivs nachvollziehbar. Schliesslich kann die Motivation der Terrorist ${ }^{*}$ innen besser verstanden werden, wenn man sich die Bedingung vergegenwärtigt, dass ein massives kollektives Leiden vorliegen muss, das mit zivilen Opfern verbunden ist und als ungerecht empfunden wird.

Günther, Ourghi, Schröter und Wiedl (2016) sehen die Anziehungskraft dschihadistischer Narrative vom Guten gegen das Böse darin, dass sie auf Missstände in islamisch geprägten Kontexten verweisen: „Dschihadistische Rechtfertigungsnarrative sind aus religiösen, politischen und sozialen Teilnarrativen zusammengesetzt, die auf bestehende Missstände verweisen und daraus ihre Überzeugungskraft gewinnen“" (2016: III). Beispiele solcher Missstände seien die Leidensgeschichten der Palästinenser oder die humanitären Katastrophen, die durch Bombardements des Assad-Regimes oder auch militärische Operationen westlicher Regierungen in Afghanistan zustande gekommen sind. Solche Teilnarrative würden als Belege eines globalen Kriegs des Westens gegen den Islam verwendet, um die Dringlichkeit der Intervention aller Musliminnen der Welt zu begründen:

Die dschihadistische Propaganda reduziert sich allerdings nicht nur auf eine Auflistung vermeintlicher Missstände, sondern bettet diese in ein geschlossenes Weltbild ein, das den als problematisch empfundenen Ist-Zustand mit einem verlockenden Zukunftsszenario kontrastiert: Die Welt unter der Herrschaft des Islams sei eine gerechte und nach den Vorgaben Gottes geordnete Welt, die den Einzelnen ein glückliches Leben beschere. (Günther et al. 2016: III)

$\mathrm{Zu}$ den wesentlichen Merkmalen dieser dualistischen Weltanschauung gehören die darin enthaltene Komplexitätsreduktion sowie deren starker Kontrast zur liberalen Moderne. Der Ohnmacht der Demokratie steht die Allmacht Gottes gegenüber; der Haltlosigkeit einer ideologieleeren Welt das feste Band zu Allah ${ }^{13}$; der frivolen, weltausbeuterischen Konsumgesellschaft das schlichte nomadische Leben des Propheten Muhammad, das in der Gestalt des saudischen Beduinen Juhayman al-Utaybi ${ }^{14}$ sowie im Leben von Osama Bin Laden in den Bergen Afghanistans einen Wiederhall findet. Je unveränderbarer die bestehende Weltordnung dargestellt wird, desto faszinierender wird der Gedanke, dass die Zuwendung an den Allmächtigen alle Probleme lösen wird.

an Apache helicopter or an F16 and fly over Tel Aviv and push a button to bomb civilians [...] Nobody would call it terrorism. Terror is always the weapon of the weak."

${ }^{13}$ Dieses von Islamisten häufig verwendetes Sinnbild entstammt dem folgenden koranischen Vers (2: 256): „Wer nicht an die Götzen glaubt, sondern an Gott, der hat den stärksten Halt ergriffen, der nicht reisst“ (Übersetzung von Hartmut Bobzin). Es ist an dieser Stelle anzumerken, dass dieser Vers in der Geschichte des Islams sehr unterschiedlich gedeutet wurde.

${ }^{14}$ Juhayman ibn Muhammad ibn Sayf al-Utaybi (1936-1980) war der Anführer der Geiselnahme in der zentralen Moschee Masjid-al-Haram in Mekka 1979. Die zentrale Forderung der von Juhayman geführten Gruppe war, dass der saudische Staat sich nach wahhabitischen Grundsätzen ausrichtet, die Gesellschaft dementsprechend umgestaltet und seine Beziehungen zum Westen abbricht. Siehe dazu Hegghammer/Lacroix (2007) sowie den Dokumentarfilm Urknall des Terrors? von Van den Berg (2018). 
Aus diesen Beobachtungen bzw. Argumenten kann hergeleitet werden, dass zumindest bestimmte dschihadistische Gruppierungen die bestehende Weltordnung sprengen wollen, damit eine neue Ordnung basierend auf islamischen Prinzipien entstehen kann. Dies erinnert an Liftons (1999) Analyse einer rechtsradikalen Gruppierung in den USA und einem Kult in Japan. In seiner Monografie „Destroying the world to save it: Aum Shinrikyo, Apocalyptic Violence, and the New Global Terrorism" zeigt er auf, dass beiden dieser Organisationen gemeinsam war, dass sie mit ihren Terroranschlägen die Rettung der Welt durch deren Zerstörung beabsichtigten.

So gesehen geht dieser Dualismus über einen schlichten, islamischen Topos hinaus, denn er dient der Konstruktion von scharfen, identitären Demarkationslinien zwischen uns, den Guten und den bösen Anderen.

Dschihadistische Gruppierungen greifen bei der Konstruktion ihrer weltanschaulichen Narrative auf wirksame Propaganda-Methoden zurück, wobei sich der sogenannte Islamische Staat (in diesem Beitrag abgekürzt als IS) hinsichtlich seiner sophistizierten und durchaus professionellen Propaganda-Methoden von allen anderen Gruppierungen unterscheidet. Zahlreiche Studien haben sich dieses Phänomens angenommen.

So analysierte beispielsweise Tirozzi (2019) 22 Videos ${ }^{15}$ des IS und konnte dabei neben der beachtlichen videotechnischen Professionalität (production quality and video styles) sieben inhaltliche Themen herausarbeiten: Freundschaft und Kameradschaft (friendship and comradery), Stärke und Sieg (strength and victory), Religion und religiöse Verfolgung (religion and religious persecution), spirituelle und existentielle Erfüllung (spiritual and existential fulfillment), Beschreibung der Gewalt (depiction of violence), Verbrechen der Feinde (crimes of the enemies) und Rufe nach Rekrutierung (calls for recruitment). Dabei unterstreicht die Autorin, dass sich die analysierten Videos viel stärker auf westliche Werte und Symbole beziehen als auf Konzepte und Doktrinen des traditionellen Islams. So schlussfolgert sie: "So, it looks like that the Islamists related with the IS, are better acquainted with the Western customs than the pan-Islamic ones, inspired by the Sunna ${ }^{16}$, approaching them through the ideological filter of the second modernity" (Tirozzi 2019: 9). Diese Art von Videoinszenierungen scheinen ein wichtiges Instrument zu sein, um Dschihadist*innen in den eigenen Ländern und jenen des globalen Nordens zu rekrutieren (siehe auch Stern/Berger 2015).

Bjørgum (2016) hebt bei der IS-Propaganda zwei Elemente hervor:

Using photos of children, who have been injured or killed in attacks from 'non-believers', IS is building up a narrative that pits Muslims against everyone else. It also speaks to a unity through race, not in the classical sense, but as a social construct of the Muslim 'race' united by their commonalities in faith. (2016: 93)

\footnotetext{
${ }^{15}$ Die Videos wurden von der IS eigenen al-Furat Media Foundation, einem webbasiertes Medienzentrum, produziert.

${ }^{16}$ Unter Sunna wird in den sunnitischen Rechtsschulen generell „die Präzedenz der Aussagen und Handlungen Muhammads und seiner Gefährten“ (Nedza 2014: 87) verstanden.
} 


\section{Zugehörigkeit zur "globalen Umma"}

Dschihadistische Gruppierungen lehnen die für muslimische Gemeinschaften typischen ethnischen Verwerfungslinien ab und orientieren sich nach einer globalen Umma. Deshalb finden die Konvertit*innen, die es in ethnisch orientierten muslimischen Gemeinschaften schwer hätten, in dschihadistischen Gruppierungen schnell Platz. Diese globale Ausrichtung ist auch für Teile der Jugendlichen anziehend, die eine Lösung länderübergreifender Missstände in antisystemischen, globalen Lösungen suchen.

Roy (2003) verweist in seiner Analyse der Radikalisierung europäischer Musliminnen zunächst einmal auf einen Wandel des Islams von einer importierten Religion - der Religion muslimischer Einwanderer - zu einer universalen Religion der in Europa geborenen oder aufgewachsenen Musliminnen. So würden sich laut Roy die Secondos nicht mehr auf den ethnisierten Glauben ihrer Eltern beziehen, sondern auf einen ethnien- und nationalitäten übergreifenden Islam. In einem zweiten Schritt würden Teile der re-islamisierten Musliminnen radikalisiert und zwar aufgrund ihrer Entfremdung vom Gastland. Es handle sich dabei meistens um junge arbeitslose Männer, die in marginalisierten Stadtteilen wohnhaft sind. Häufig bedeutet die Re-Islamisierung auch eine Abkehr von einem Lebensstil, der als verwestlicht, hedonistisch und frivol abgestempelt wird.

Hinweise auf die Zugehörigkeit zu einer Umma, die ethnische, sprachliche und nationale Unterschiede überwindet, sind in den Narrativen der Dschihadist*innen allgegenwärtig, wie das folgende Beispiel par excellence aufzeigt:

[Now] every day there are not only muhajirin ${ }^{17}$ to the land of Islam but also muhajirat ${ }^{18}$ who were sick of living amongst $k_{u f r}{ }^{19}$ and its people. As soon as the sun of their awaited state rose, they rushed to it alone and in groups from the eastern and western extents of the Earth. Their colors and tongues are different, but their hearts are united upon 'there is no god but Allah. ${ }^{20}$ I remember the day I performed hijrah $^{21}$, I was the only Arab woman amongst the muhajirah ${ }^{22}$ sisters during that trip. (Al-Muhajirah 2015; zit. nach Bjørgum 2016: 96)

Dieses Beispiel zeigt deutlich, wie der Anschluss an den IS den Dschihadistinnen ein Gefühl der globalen Zugehörigkeit verleiht und ihnen durch einen heiligen Lebenszweck und Solidarität eine befriedigende Selbsterfahrung ermöglicht.

\footnotetext{
${ }^{17}$ Das arabische Wort muhajerin ist der Plural von muhajir (Auswanderer). Gemeint ist hier die Auswanderung in die Domäne des Islams.

${ }^{18}$ Das arabische Wort muhajirat ist die weibliche Form für Muhajerin.

${ }^{19}$ Das arabische Wort kufr bedeutet Unglaube. Hier ist „die Domäne der Ungläubigen“ - nämlich der globale Norden gemeint.

${ }^{20}$ Das ist die Übersetzung des arabischen Satzes la ilaha illallah, eines Teils der islamischen Glaubensbekenntnis, der in den täglichen Gebeten mehrmals vorkommt.

${ }^{21}$ Das arabische Wort hijra (Migration/Auswanderung) ist eine Anspielung auf die Flucht des Propheten Muhammad von Mekka nach Media im Jahr 622. Dieses Jahr gilt als der Beginn der islamischen Zeitrechnung.

${ }^{22}$ Das arabische Wort muhajirah bedeutet Migration.
} 


\section{Fazit}

In diesem Abschnitt konnten verschiedene Facetten der kollektiven Identität im Kontext des Dschihadismus dargelegt werden. Zunächst einmal wurde auf die im Entstehen begriffenen, islamisch artikulierten kollektiven Identitäten in der islamischen Welt bis in die 1980er Jahre verwiesen. Sodann wurde aufgezeigt, wie diese kollektiven Identitäten von neuen kollektiven Selbst- und Weltbildern überschattet wurden, die auf einer dualistischen Weltanschauung im Sinne der Dichotomie zwischen dem Guten und dem Bösen basieren. Schliesslich wurde dargelegt, wie Umma zu einer länderübergreifenden imagined commuinty (Anderson 1983) hochstilisiert wurde.

\section{Relevanz der Identität auf der Mesoebene}

Wie bereits erwähnt, ergeben sich bestimmte Facetten dschihadistischer Identitäten aus der Interaktion zwischen zum Dschihadismus geneigten Individuen und dschihadistisch gesinnten Gruppierungen. Dabei scheinen die folgenden zwei Phänomene von besonderer Bedeutung zu sein: a) identitäre Beeinflussung der neuen Dschihadistinnen durch deren Rekrutierer; und b) Gruppenzugehörigkeit.

\section{Identitäre Beeinflussung}

Berücksichtigt man den radikalen Sinneswandel von Individuen, die von dschihadistischen Gruppierungen rekrutiert werden, liegt die Annahme nah, dass dschihadistische Ideologen und Prediger über wirksame Techniken der Indoktrinierung von Menschen verfügen. Denn nur so kann die Umwandlung von mehr oder weniger unauffälligen, teilweise sogar gut integrierten Individuen, zu eifrigen Selbstmordattentätern oder sogar hemmungslosen Henkern, die ihre Opfer durch archaische Methoden vor laufende Kameras hinrichten (wie dies beispielsweise beim IS der Fall war), verstanden werden.

Stahelski (2005) zufolge indoktrinieren Terroristenzellen generell ihre Mitglieder in ähnlicher Art und Weise wie bei einem Kult ${ }^{23}$ und zwar durch ein Prozedere, das mehrere Phasen durchläuft:

In der ersten Phase (depluralization phase) trennt die Zelle ihre Mitglieder von ihrem sozialen Umfeld und schwächt deren multiplen Zugehörigkeiten. Es soll am Ende die Zugehörigkeit zur Zelle als der einzig relevante soziale Bezug übrigbleiben. Danach wird in der zweiten Phase (self-deindividuation phase) die Individualität der Mitglieder beseitigt, sodass alle möglichst gleichgeschaltet sind. In der dritten Phase (other-deindividuation phase) werden die Gegner, durch Aberkennung ihrer Individualität, zu blossen Mitgliedern eines bösen Kollektivs reduziert. Sind einmal die Gegner keine Individuen mehr, wird ihnen in der vierten Phase (dehumanization phase) auch die Menschlichkeit aberkannt. Schliesslich werden die deindividualisierten und entmenschlichten Gegner in der fünften Phase (demonization phase) auch noch dämonisiert (siehe auch Rodríguez-Carballeira/Martín-

\footnotetext{
${ }^{23}$ Stahelski verwendet das englische Wort sect. Da aber das deutsche Wort Sekte nicht genau dieselbe Bedeutung hat und zudem nicht werteneutral ist, wurde hier sect mit Kult übersetzt.
} 
Peña/Almendros/Escartín/Porrúa/Bertacco 2010). Wie gross das Erklärungspotential dieses Ansatzes für die gewalttätigen Handlungen der dschihadistischen Organisationen ist und ob er bei allen dieser Organisationen gleichermassen Gültigkeit besitzt, ist noch nicht ausreichend untersucht worden. Dennoch gibt es einige Publikationen zu dieser Thematik, inklusive der beiden Folgenden, die in ihrer Logik diesem Ansatz nahekommen.

Aus einer security studies Perspektive, und deshalb nicht ganz wertneutral, beschreibt Gaub (2016) in einem Essay die Techniken, durch welche der IS ihre Mitglieder rekrutiert, deren Denkweise beeinflusst und sie somit zu gewalttätigen Handlungen animiert oder sogar zwingt.

Des Weiteren versucht Murawiec (2008) in seinem Buch The Mind of Jihad durch eine Collage aus historischem Material aufzuzeigen, dass der modern jihad (so seine Bezeichnung für Dschihadismus) einen Kult der Gewalt und Macht darstellt, die, ähnlich wie mittelalterliche Millenarier und Apokalyptiker in Europa, sich einer gnostischen Ideologie bedient, um ihre Mitglieder mental zu kontrollieren und zu Gewalthandlungen zu bewegen. Des Weiteren argumentiert Murawiec, dass die politischen Technologien dschihadistischer Gruppierungen grosse Ähnlichkeiten mit denjenigen der Bolschewiki aufweisen. Während bei solchen QuerVergleichen höchste Vorsicht geboten ist, enthält das Buch dennoch einige gute Anregungen für die Dschihadismus-Forschung.

\section{Gruppenzugehörigkeit}

Eine zentrale, für den Dschihadismus relevante Facette der Identität auf der Meso-Ebene ist die Gruppenzugehörigkeit, die in der Tradition von Tajfel und Turner (1986) als social identity bezeichnet wird. Verschiedene Studien haben die Relevanz dieser Form der Identifikation für Dschihadismus hervorgehoben. Frindte et al. (2016) etwa haben darauf verwiesen, dass die zum Dschihadismus geneigten Individuen in salafistisch/dschihadistischen Milieus ein soziales Stütz- und Bezugssystem finden, was besonders in der Einstiegsphase ihrer dschihadistischen Karriere von grosser Bedeutung ist:

Zugehörigkeit, ein Gefühl von Akzeptanz und emotionaler Rückhalt in der Gruppe stellen relevante motivische Antriebsfedern für das Eintauchen in die extremistischen Umfelder und für gruppenkonformes Verhalten dar. Im Zuge der Gruppenzugehörigkeit wächst dann auch die Identifikation mit den religiös verbrämten Ideologieangeboten. Gruppendynamische Prozesse und extremistische Diskurse, sowohl in der realen als auch in der virtuellen Welt, können derartige Identifikationen legitimieren und verstärken. (Frindte et al. 2016: 31)

Hinweise auf die Bedeutung der Gruppenzugehörigkeit für den Anschluss an militante Gruppierungen kommen aber auch aus den Studien, die sich nicht spezifisch mit Dschihadismus befassen. Beispielsweise haben Haddad Kreidie und Renwick Monroe (2002) in ihren Interviews mit Mitgliedern verschiedener verfeindeter Gruppierungen im libanesischen Bürgerkrieg (1975 bis 1990) aufgezeigt, dass es vor allem eine starke Identifikation mit einer ethnisch-religiösen Gruppe und die Wahrnehmung einer Bedrohung dieser Gruppenidentität durch Outgroups war, welche die Interviewten zur Teilnahme am Bürgerkrieg bewegt hat. 
Aus der Gruppenzugehörigkeit geht in der Regel auch eine intensiv gelebte Gruppensolidarität und Kameradschaft hervor, die sich nicht selten zu einer Eigendynamik entwickeln kann. Dies kann so weit gehen, dass die ideologische Zielsetzung durch Dringlichkeit des Gruppenschutzes bzw. der Rache für die Kameraden überschattet wird (siehe etwa Atran 2010: xi).

\section{Fazit}

Wie bereits aufgezeigt, vermitteln dschihadistische Strukturen auf der Mesoebene (Milieus, Netzwerke, Zellen usw.) den (neu eingestiegenen) Dschihadit*innen Orientierung sowie Gefühle der Zugehörigkeit. Zudem verfügen dschihadistische Zellen, ähnlich wie bei einem Kult, offenkundig über wirksame Techniken, um die Identität der Menschen nach ihren Vorstellungen zu beeinflussen.

\section{Relevanz der Identität auf der Mikroebene}

Obwohl die Identität auf der Mikroebene - im Sinne individueller Selbsterfahrung - für den Dschihadismus in vielerlei Hinsicht relevant zu sein scheint, fokussiert sich dieser Abschnitt auf drei Facetten dieses Phänomens, die in der Forschung grössere Beachtung erfahren haben. Dies sind: a) Adoleszenz, weil diese Lebensphase mit Identitätswandel einher zu gehen scheint, wodurch die Jugendlichen für dschihadistische Ideologien empfänglicher werden; $b$ ) $(\mathrm{Re})$ Konversion, weil Dschihadist*innen im globalen Norden entweder Konvertit ${ }^{\star}$ innen sind oder Rekonvertit*innen (bzw. born-again Musliminnen), und weil (Re)Konversion auch einen Identitätswandel bedeutet (Travisano 1970; Gooren 2010: 46; Sheikhzadegan 2020); und c) Agency, weil die neuen Dschihadist*innen bei der Neuorientierung ihres Selbst aus ihrem gewohnten Alltag ausbrechen, um aktiv in das Weltgeschehen einzugreifen.

\section{Adoleszenz und die Suche nach Sinn}

Das psychosoziale Moratorium im Prozess des Erwachsenwerdens macht die Jugend für vielfältige Weltdeutungsangebote anfällig. Jugendliche im Teenager-Alter sind besonders beeinflussbar und können somit leichter von erfahrenen Ideologen rekrutiert werden:

We know that adolescence and youth is a critical period, characterised by transition, formation, and growing up; a period when the individual must adjust and adapt to his/her social environment, and seek and attempt to consolidate his/her own identity. It is, therefore, a stage during which the individual is more vulnerable to external sources of influence or pressure. (Rodríguez-Carballeira et al. 2010, o.S.)

In einem theoretischen Aufsatz ist Meeus (2015) der Frage nachgegangen, weshalb junge Menschen im globalen Norden sich dschihadistischen Gruppierungen anschliessen. In An- 
lehnung an die Theorie des Identitätsstatus von Marcia (1966) ${ }^{24}$ sowie das Stufenmodell der religiösen Konversion von Lofland und Stark (1965) argumentiert Meeus, dass Jugendliche, die eine instabile Identität aufweisen (die Typen diffusion und moratorium im Marcias Modell), leichter als andere Jugendliche von radikalen Gruppierungen rekrutiert werden können. Erfüllen diese Jugendlichen auch noch die Bedingungen des Modells von Lofland und Stark (sie leiden unter anhaltenden Spannungen; sie sind zur Ansicht gelangt, dass die Lösung ihres Unbehagens in einer Religion zu finden ist; ihnen fehlen soziale Bindungen, die sie durch diese Krise begleiten; und sie kommen in zunehmenden Kontakt mit einer dschihadistischen Gruppierung), so können sie leichter von diesen beeinflusst werden als andere Jugendliche:

Young people with poor family relationships and poor friendships are especially likely to lack appropriate interpersonal contexts for building an identity. A new group with a clear, defined mission may therefore be able to solve their problems in two ways: it offers warm interpersonal bonds, as well as potential new personal goals and commitments. Groups of jihadists are perfectly fit to serve this twofold purpose. Without any doubt, groups have the capacity to install new identities in their members, as Lofland and Stark showed. (Meeus 2015: 279)25

$\mathrm{Zu}$ den wichtigen Aspekten der Identitätssuche gehört das Streben nach einem Lebenszweck. In zahlreichen Narrativen der Dschihadist*innen kommt die Betroffenheit über das Los der unterdrückten Musliminnen, etwa in Palästina, Irak, Syrien oder Jemen, stark zum Ausdruck. Hier ein Beispiel: In seiner autobiografischen Erzählung berichtete ein Schweizer Konvertit und Dschihad-Reisender (Eser Davolio/Banfi/Gehrig/Gerber/Luzha/Mey/Möwe/Müller/Steiner/Suleymanova/Villiger/Wicht 2015: 9), dass er zunächst aus seiner grossen Sympathie mit dem Los der Palästinenser heraus nach Palästina reiste, um als Ambulanzfahrer den kämpfenden Palästinensern zu helfen. Danach zog er aus Gründen der Solidarität mit dem IS nach Syrien.

\section{(Re-)Konversion}

Dschihadist ${ }^{\star}$ innen im globalen Norden bestehen in der Regel entweder aus Konvertit ${ }^{\star}$ innen oder Re-Konvertit ${ }^{\star}$ innen (Roy 2007: 52). Die Letzteren sind Musliminnen, die zwar als solche geboren wurden, die jedoch durch einen Wandlungsprozess eine neue Sicht auf ihre Religion entwickelt haben. (Re-)Konversion geschieht nicht plötzlich, sondern in einem Prozess, den Paloutzian (2005) als spirituelle Transformation beschrieben und wie folgt definiert hat: „[...] a spiritual transformation constitutes a change in the meaning system that a person

\footnotetext{
${ }^{24}$ Gemäss dieser Theorie kann die Identität eines Menschen in der Adoleszenz vier Stati annehmen: Identity Diffusion (Identitätsdiffusion: Das Individuum befasst sich (noch) nicht mit der Frage, was es in der Zukunft werden möchte), Identity Foreclosure (Identitätsübernahme: Das Individuum hat sich bereits entscheiden, die künftige Rolle, die seine Bezugspersonen ihm wünschen, zu übernehmen), Identity Moratorium (Moratorium: Das Individuum sucht selber nach einer künftigen Rolle und erlebt deshalb eine Identitätskrise), und Identity Achievement (erarbeitete Identität: Das Individuum hat das Moratorium hinter sich gebracht und sich zu einer künftigen Rolle verpflichtet) (für eine Kritik dieser Theorie siehe Kraus/Mitzscherlich 1997).

${ }^{25}$ Studien über andere Formen von gewalttätigen, politischen Bewegungen heben ebenfalls den Faktor Adoleszenz hervor (siehe etwa Veelken 1978; Pilz 1994).
} 
holds as a basis for self-definition, the interpretation of life, and overarching purposes and ultimate concerns [...]“ (Paloutzian 2005: 334).

Durch einen solchen Sinneswandel entwickeln (Re-)Konvertit*innen also ein neues Selbstund Weltverständnis und deuten somit den Sinn und Zweck ihres Lebens neu.

Bei ihrer Analyse der Entwicklung des neuen Glaubens nach der Konversion zum Islam hat Roald (2012) aufgezeigt, dass Konvertit*innen zu Beginn ihrer Bekehrung häufig sehr eifrige Muslim*innen und sozusagen more loyal than the king werden. In Anlehnung an Susmans (1979) dichotomes Persönlichkeitsmodel (character vs. personality) ${ }^{26}$ postuliert Roald, dass Europäer*innen durch Konversion zum Islam einen Wandel vom Modus personality (basierend auf Selbstverwirklichung) zum Modus character (basierend auf Selbstaufopferung) durch machen.

Rushchenko (2017) hat aufgezeigt, dass Konversion zwar die Konvertit*innen anfällig für Extremismus macht, dass jedoch Konversion erst durch Kombination mit anderen Faktoren wie kriminelle Vergangenheit, Stigmatisierungserfahrungen, Absenz der Eltern, Identitätskonflikte und Beeinflussung durch radikale Prediger - zum Dschihadismus führt.

Nollert (2019) hat darauf verwiesen, dass unter den Dschihadist*innen im globalen Norden eine auffällig grosse Zahl von Konvertit*innen zu finden ist. Dieses Phänomen lässt sich ihm zufolge dadurch erklären, dass die gewaltbereiten Konvertit ${ }^{\star}$ innen meistens sehr junge Menschen sind, die im Islam nicht primär Spiritualität und Barmherzigkeit, sondern Regeln und eine Abkürzung ins Paradies suchen. Zudem erzählen sie meistens von einer schwierigen Vergangenheit:

Ihre Lebensläufe und autobiografischen Narrative verweisen [...] häufig auf familiäre, schulische und berufliche Probleme, religiöse und bildungsferne Herkunftsmilieus, Drogenkonsum, Delinquenz, Haftstrafen, Sinnprobleme und die Sehnsucht nach einer von Freundschaft und gegenseitiger Anerkennung geprägten Heimat. (Nollert 2019: 17)

Gemäss Nollert soll die starke Vertretung der Konvertit*innen unter den Dschihadist*innen auch dadurch erklärt werden, dass sie regen Kontakt zu den Salafist*innen hatten.

Nach ihren Analysen der radikalen, in Grossbritannien angesiedelten Organisation alMuhajiroun, argumentieren Wiktorowicz und Kaltenthaler (2006), dass radikale islamistische Gruppierungen durch Anbieten selektiver jenseitsbezogener Anreize Musliminnen mit starker religiöser Überzeugung in ihren Bann ziehen. Zwar überwiegen bei manchen Individuen nicht-religiöse Motive, wie etwa Rachegelüste, das Gefühl der Ermächtigung (Empowerment) oder Durst nach Anerkennung. Bei vielen anderen überwiegen jedoch religiöse Motive:

In cases where individuals take spirituality seriously, movement ideologies offer strategies for fulfilling divine duties and maximizing the prospects of salvation on

\footnotetext{
${ }^{26}$ Gemäss dem Kulturhistoriker Susman (1979) erlebte die US-amerikanische Gesellschaft Anfang des 20. Jahrhunderts einen kulturellen Wandel, in dem Menschen sich vom Typus character (introvertiert, moralisch, selbstbeherrschend, sich aufopfernd) zum Typus personality (extrovertiert, auf Selbstdarstellung orientiert, nach Bestätigung strebend, empfänglich für Werbung und Celebrity-Kultur) verwandelten.
} 
judgment day. In essence, these ideologies serve as heuristic devices or templates that outline the path to salvation. Where individuals believe that the spiritual payoffs outweigh the negative consequences of strategies in the here and now, high-cost/risk activism is intelligible as a rational choice. (Wiktorowicz/Kaltenthaler 2006: 295296)

Zwar sehen die Autoren in diesen Befunden Belege für die Relevanz des rational choice Ansatzes. Identitätstheoretisch lässt sich aber genauso gut argumentieren, dass die betreffenden Dschihadist*innen im Einklang mit ihrem Selbstverständnis als gläubige Menschen handeln. Mit anderen Worten, sie entscheiden sich für Dschihadismus, um die Authentizität ihres pious self (Jouili/Amir-Moazami 2006) zu wahren.

\section{Agency}

$\mathrm{Zu}$ den vielen Facetten der Identität gehört auch die Agency. ${ }^{27}$ Verschiedene Studien zeigen unter den Dschihadist*innen eine erhöhte Agency und ein Streben danach, das eigene Schicksal selber in die Hand zu nehmen. Lindemann (2018) etwa befragte dreizehn mutmassliche Mitglieder der al-Qaida und des IS in französischen Gefängnissen. Aus den Interviews geht hervor, dass die Faszination der Befragten für Dschihadismus durch deren Streben nach Anerkennung zu erklären sei, wobei nicht ein höherer Status im Vordergrund stehe, sondern der Wunsch danach, eine Verbesserung der Situation der Musliminnen zu bewirken. Somit sei militanter Aktivismus (auch) als eine Überwindung von aberkannter Agency zu deuten.

In ihrer ethnografischen Studie über Dschihadistinnen zeigte Casutt (2018) auf, dass diese, entgegen der allgemeinen Annahme, keine passiven, sondern aktive Individuen mit einer starken Agency sind (siehe hierzu auch Parashar 2010). Zudem unterstreicht sie, dass die Teilnahme von Frauen am Dschihad eher ihrer religiösen Überzeugung entspringt als ihrer Emotionalität.

In ihrer Diskursanalyse einer Auswahl von Medienberichten über drei Gruppen von Frauen, nämlich denjenigen, die sich dem IS anschliessen, denjenigen, die dem IS zum Opfer fallen, und denjenigen, die den IS bekämpfen, schlussfolgert Sjoberg (2018), u.a., dass die Agency-Frage bei den weiblichen Helfern wie auch Opfern des IS beinahe aberkannt und bei Frauen, die den IS bekämpfen, überschätzt wird.

Der Frage der Agency kommt auch im Kontext der Globalisierung eine besondere Bedeutung zu. Aufgrund der global agierenden Massenmedien werden die Nachrichten über die Missstände in der islamischen Welt heute viel schneller und effizienter zirkuliert, als dies früher der Fall war. Da keine der vorhandenen Ideologien adäquate und nachhaltige Lösungen für sämtliche dieser Krisen zu bieten scheint, ist in letzten Jahrzehnten ein ideologisches Va-

\footnotetext{
${ }^{27}$ Diesbezüglich beziehe ich mich auf die temporale Dimension narrativer Identität nach Lucius-Hoene und Deppermann (2004:59-60). Diese dreht sich um die Frage, wie der Erzählende seine/ihre Biografie in zeitlicher Hinsicht strukturiert. $\mathrm{Zu}$ verschiedenen Aspekten der temporalen Dimension gehört auch die Agency, nämlich die Frage, „ob und in welchen Aspekten und Bereichen seines Lebens er sich als handelnde Person, als Zentrum der Geschehnisse seines Lebens, als Inhaber von Kontrollmöglichkeiten und Entscheidungsspielräumen erlebt, oder ob und hinsichtlich welcher Erfahrungen er sich von heteronomen Mächten dirigiert fühl“" (59). Für die besondere Relevanz eines Selbstbildes als aktiv agierender Mensch im Kontext der sogenannten Postmoderne siehe Eickelpasch/Rademacher (2015).
} 
kuum entstanden. Das Gefühl der Ohnmacht ist eine Einladung für eine ganzheitliche und radikale Ideologie, die einen systemischen Umbau der Weltordnung verheissen würde.

Mit dem Hinweis auf westliche Konvertit ${ }^{*}$ innen, die sich einer dschihadistischen Bewegung angeschlossen haben, schreibt Roy (2003, o.S.):

Twenty years ago such individuals would have joined radical leftist movements, which have now disappeared or become "bourgeois"[...] Now only two Western movements of radical protest claim to be "internationalist": the anti-globalization movement and the radical Islamists. To convert to Islam today is a way for a European rebel to find a cause; it has little to do with theology. (Roy 2003: o.S.)

Beispielswiese verweist der Autor auf den französischen Konvertiten Lionel Dumont, der zum Islam konvertiert sei, weil es nur der Islam sei, der heute gegen das System kämpft. Aufgebracht durch die ethnische Säuberung der Musliminnen in Bosnien kämpfte er in Bosnien Seite an Seite mit diesen Menschen.

\section{Fazit}

Wie in diesem Abschnitt gezeigt wurde, kommen bei der Genese dschihadistischer Identitäten auf der Mikroebene verschiedene Facetten der Identität zum Tragen.

Menschen in der Adoleszenz sind häufig Identitäts- und Sinnkrisen ausgesetzt, weshalb sie für Sinnangebote empfänglicher als andere Altersgruppen zu sein scheinen. Menschen können sich auch vom Dschihadismus angezogen fühlen, wenn sie durch einen Sinneswandel den Islam annehmen (Konversion) oder neu „entdecken“ (Rekonversion). Schliesslich ist die Agency im Sinne des Selbstbildes als aktiver Mensch insofern relevant, als Dschihadist*innen ihr Schicksal selber in die Hand nehmen und aktiv in das Weltgeschehen eingreifen möchten.

\section{Multidimensionale Ansätze/Studien}

In diesem Abschnitt werden nun Studien besprochen, die bei ihren Analysen des Dschihadismus gleich mehrere Ebenen oder Facetten der Identität berücksichtigt haben. Dabei sollen auch Studien berücksichtigt werden, die zwar die Frage der Identität nicht systematisch oder explizit angehen, die dennoch mehrschichtige, identitätstheoretisch relevante Anhaltspunkte liefern.

\section{Profil der Dschihadist*innen}

Zwar konnte bislang kein einheitliches Profil der Dschihadist*innen erstellt werden (McGilloway/Ghosh/Bhui 2015; Eser Davolio et al. 2015; Hafez/Mullins 2015: 959. Siehe auch Bornschier 2007: 415-416), dennoch haben sich einige Forschende es mit Typologien versucht. Im Folgenden werden exemplarisch zwei vorgeschlagene Typologien skizziert.

Nesser (2010) konnte anhand seiner Analyse von zwei Dschihadi-Zellen in Europa folgende Persönlichkeitstypen identifizieren: 
Die entrepreneurs (Anführer) sind Idealisten mit einer ausgesprochenen Führungsgabe. Sie sind gut integrierte Bürger, die sensibel auf die Leiden ihrer Glaubensbrüder reagieren und deshalb auf Gewalt zurückgreifen. Die protégés (Jünger) oder junior entrepreneurs folgen ihren Anführer*innen auf Schritt und Tritt. Sie sind Idealisten, gut integrierte Bürger, scharfsinnig, gebildet, rational denkend und oft virtuose Techniker, jedoch jung, unerfahren und leicht beeinflussbar. Die misfits (die Sozialfälle) sind Individuen mit einer schwierigen Vergangenheit. Gezeichnet durch soziale Probleme suchen sie nach Halt und finden ihn im Dschihadismus, was ihnen zugleich einen Ausweg aus ihrer Misere und einen Neuanfang ermöglicht. Schliesslich sind die drifters (die Labilen) weder sonderlich religiös noch politisch, noch interessieren sie sich für eine bestimmte Ideologie, noch leiden sie unter gravierenden sozialen Problemen. Sie sind DschihadistInnen geworden, weil sie mit Dschihadist ${ }^{\star}$ innen im Kontakt standen und von diesen rekrutiert wuden.

Was die Identitätsprofile dieser vier Persönlichkeitstypen anbelangt, lassen sich die entrepreneurs als Menschen mit einer starken Agency und einem altrusitischen Selbstbild bezeichnen, die protégés als Menschen mit einem starken Bedürfnis nach der Akzeptanz durch ihre signifikanten Anderen, die misfits als Menschen mit angeschlagener Selbstachtung und die drifters als Menschen ohne einer konsolidierten Identität.

Beim zweiten Beispiel handelt es sich um die von Khosrokhavar (2009: 233-237) vorgeschlagene Typologie. Ihm zufolge lassen sich unter den Dschihadist ${ }^{\star}$ innen fünf Persönlichkeitstypen identifizieren: The missionary ist der Missionar, der andere Musliminnen dazu einlädt, für die Errichtung einer islamischen Ordnung, die sie als die vollkommenste erachten, $\mathrm{zu}$ kämpfen. The macho hält moderne Geschlechterverhältnisse für dekadent und ist der Überzeugung, dass nur durch Gewalt die heile patriarchale Ordnung wiederhergestellt werden kann. The upholder of justice glaubt daran, dass die islamische Welt vom westlichen und vor allem US-amerikanischen Imperialismus unterjocht wird und dass nur Dschihad sie aus dieser Misere befreien kann. The adventurer ist der Abenteurer, der Freude an der Entdeckung der Welt sowie der Geselligkeit mit aussergewöhnlichen Menschen hat und die führenden Dschihadist ${ }^{\star}$ innen wie Kultfiguren bewundert. The existential man fühlt sich von der Gesellschaft nicht ernst genommen bzw. respektiert und findet im Dschihad eine Restaurierung seiner Selbstachtung.

Aus diesen Beschreibungen lassen sich folgende Identitätsprofile für diese fünf Persönlichkeitstypen rekonstruieren: Der missionary entspricht mehr oder weniger dem Profil der entrepreneurs bei Nesser. Der upholder of justice dürfte über ein sehr moralisches wie auch kämpferisches Selbstbild verfügen. Beim macho lässt auf ein ausgeprägt maskulines wie auch traditionsbewusstes Selbstverständnis schliessen. Der adventurer ist um eine Neupositionierung seines Selbst in der Welt bemüht und der existential man erinnert an das Profil der misfits bei Nesser.

\section{Vielschichtige Selbstbilder der Selbstmordattentäter}

Um den subjektiven Sinn von dschihadistischen Selbstmordattentaten zu verstehen, analysierte Schulze (2007) Kommuniqués der Dschihadist*innen, welche zwischen 1995-2006 erschienen sind. Er schlussfolgerte, dass gewalttätige Handlungen der Dschihadist*innen auf folgenden Gültigkeitsbehauptungen beruhen: 
Die Tat ist die Vollstreckung von Gerechtigkeit.

Die Tat ist der Ausdruck einer kritischen Handlung, die äquivalent zum Gebet zu verstehen ist.

Die Tat ist zwingend, da sie der Ausdruck einer beginnenden Apokalypse ist.

Die Tat ist Seinsausdruck des Handelnden, durch sie erfährt er radikal seine Existenz.

Die Tat ist im Falle des Selbstmordes die höchste und letztmalige Form der ExistenzErfahrung.

Die Tat ist Ausdruck eines externen Willens, dessen Vollstrecker der Handelnde ist.

Die Tat ist moralisch gut, da sie das moralisch Gute gegen das Böse setzt.

Die Tat ist geschichtlich, da sie Teil eines generalisierten Konfliktes zwischen zwei Welten („Zivilisationen“) ist. (Schulze 2007: 86)

Aus dieser Studie ergibt sich gleich ein vielschichtiges, identitätstheoretisch relevantes Bild, denn darin lassen sich zugleich Anhaltspunkte für eine Verortung des Selbst in der kosmischen Ordnung (Vollstrecker Gottes Willen, Apokalypse), Verortung in der Geschichte (Konflikte zwischen zwei Welten), Verortung in einer normativen Ordnung (das Gute vs. das Böse, die Gerechtigkeit), ein spirituelles Selbstbild und eine intensive Selbsterfahrung finden.

\section{Zusammenspiel von verschiedenen Identitätsebenen}

Das vom IS konstruierte, dichotome Weltbild wird auf der Mikroebene in unzähligen Narrativen der Dschihadist*innen, die sich dem IS angeschlossen haben, wiedergespielt. Was die jihadi brides anbelangt, kommt in ihren Blogs, so Bjørgum (2016), neben der Romantik der hijra (Migration) aus dem dar-al-harb - Haus des Krieges, der Region von kufr (Unglaube) in das gelobte dar-al Islam (Haus des Islams, Regionen, in denen das Gesetz des Islams vorherrscht) auch noch die Romantik der Eheschliessung mit einem Dschihadisten hinzu: „The romantic vision of an Islamic utopia, combined with the promise of a romantic partner at the end of the road is strong in these narratives" (Bjørgum 2016: 96).

Hemmingsen (2010) ist bei ihrer Analyse dreier dschihadistischer Zellen in Dänemark der Frage nachgegangen, wieso diese Ideologie für die betreffenden Dschihadist*innen anziehend war. Sie konnte dabei drei Typen von Attraktion dieses Phänomens für junge Menschen identifizieren:

Individuelle Attraktion bedeutet, dass Dschihadismus als eine Gegenkultur wirkt und deshalb aus vier Hauptgründen junge Mensch in seinen Bann ziehen kann: Tatendrang (action), Zugehörigkeitsgefühl (social belonging), intellektuelle Herausforderung (intellectual challenges) und trotziges Verhalten (being counter). Kontextabhängige Attraktion bezieht sich auf den Umstand, dass der Dschihadismus in der Gegenwart aus zwei Gründen anderen Gegenkulturen überlegen ist. Er ist hochbrisant (high-profiled); und er gilt als eine Gefahr für die Sicherheit, weshalb die Dschihadist ${ }^{\star}$ innen ernst genommen werden. Mit spezifischer Attraktion meint sie schliesslich, dass Dschihadismus attraktiv wirkt, weil er Individuen zum Ausbruch aus den Fesseln der lästigen sozialen Erwartungen, Normen und Bräuchen animiert und ihnen ein Gefühl der Agency vermittelt. 


\section{Verschiedene Identitätsdimensionen aus der Rational-Choice-Perspektive}

Pisoiu (2012) hat sich aus der Warte des Rational-Choice-Ansatzes mit der Frage befasst, durch welchen Prozess die Menschen zu Dschihadist*innen werden. Zunächst einmal unterstreicht sie, dass Dschihadist*innen sich von anderen Menschen nicht unterscheiden und, dass der Dschihadismus lediglich durch ein Labelling, welches im Kontext von MajoritätMinorität-Beziehungen zustande kommt, zum devianten Verhalten erklärt wird. Somit wiederspiegele dieses Labelling eher die Sicht der Majorität und nicht diejenige der Dschihadist ${ }^{*}$ innen, die sich eher als Freiheitskämpfer und Revolutionäre ansehen. Sodann verweist sie darauf, dass man nicht über Nacht zum Dschihadisten wird, sondern im Lauf eines Prozesses, der einer Berufskarriere ähnelt. Dieser Prozess basiere auf der rationalen Erwägung von drei Faktoren: Standing, Recognition und Reward. Beim Standing handelt es sich um die Übernahme von Rollen, die im relevanten sozialen Umfeld als positiv geschätzt werden. Bei Dschihadist*innen basieren solche Rollen auf Werten wie Mut, Altruismus, Macht und spezialisiertem Wissen. Recognition bezieht sich auf die Anerkennung einer DschihadistenKarriere im relevanten sozialen Umfeld; und unter Reward versteht die Autorin den Drang, etwas gegen eine soziale Misere zu unternehmen (to make a difference) (Pisoiu 2012).

Dieser Ansatz ist hinsichtlich der dschihadistischen Identitäten insofern aufschlussreich, als er das dschihadistische Engagement mit verschiedenen Facetten der Identität, nämlich der Übernahme von neuen sozialen Rollen, dem Streben nach der Anerkennung und der Entwicklung eines altruistischen Selbstverständnisses, in Verbindung bringt.

Um es zusammenzufassen, konnten aus den in diesem Abschnitt besprochenen Studien Anhaltspunkte für verschiedene Facetten der Identität gewonnen werden. Erstens haben wir gesehen, dass die Suche nach einem einheitlichen Profil der Dschihadist ${ }^{\star}$ innen deshalb nicht möglich ist, weil es sich dabei um sehr unterschiedliche Persönlichkeitstypen handelt. Zweitens gewannen wir einen Einblick in die vielschichtigen Selbstbilder der Selbstmordattentäter. Drittens konnte aufgezeigt werden, wie dschihadistische Narrative individuell rezipiert und bearbeitet werden. Schliesslich wurden die aus einer Rational-Choice-Perspektive hervorgehenden, identitätsrelevanten Anhaltspunkte dargelegt.

\section{Schlussfolgerungen}

Dschihadismus - als Sammelbegriff - basiert auf einer apokalyptischen Ideologie, welche das Heil der islamischen Welt darin sucht, sie nach islamischen Prinzipien, wie auch immer diese zu verstehen sind, umzubauen. Ein solches Unterfangen setzt, so das Narrativ, einen epischen Kampf zwischen dem Guten und dem Bösen voraus, für welchen jede Form von Aufopferung gerechtfertigt ist.

Als Konkurrent und teilweise Ablöser anderer radikaler Ideologien liefert der Dschihadismus eine Welttheorie, welche die gesamte Weltgeschichte komplexitätsreduzierend in einen kosmischen Gesamtplan stellt, der vom Allmächtigen entworfen und realisiert wird. Die Missstände in der islamischen Welt werden auf das Wirken heimtückischer politscher Regimes zurückgeführt, die es zu besiegen und zu zerschlagen gilt. Dschihadist ${ }^{\star}$ innen sehen sich als Instrumente Gottes bei der Verwirklichung dieses „heiligen“ Plans. Was am Ende dieses grossen Kampfes entstehen soll, ist die Wiederherstellung des Früh-Islam, wie er, gemäss 
Dschihadist*innen, von den al-salaf al-salih (den frommen Altvorderen) verstanden und gelebt wurde.

So schlicht dieses Narrativ auf der Makroebene ist, so vollzieht sich die Bekehrung zum Dschihadismus auf der Meso- und Mikroebene auf verschiedene Wege und durch eine Vielzahl von Mechanismen, wie etwa Sinnsuche, Streben nach Anerkennung, Integrität eines rigiden pious self, Suche nach Gemeinschaft und Zugehörigkeit, Traumabewältigung, Restaurierung einer angeschlagenen Selbstachtung, identitäre Beeinflussung (Indoktrinierung), Hang zum Radikalismus, Hang zur Rebellion - being counter (Hemmingsen 2010) -, Suche nach einem Lebenszweck, Identifikation mit den Kriegsopfern bzw. mit der globalen Umma, aber auch Abenteuerlust oder Rachegelüste. Hervorzuheben ist dabei die starke Präsenz Jugendlicher in dschihadistischen Bewegungen - ein Phänomen, das auf der Mikroebene auf die Empfänglichkeit bestimmter Gruppen von jungen Menschen für eine auf dem radikalen Umbau der (islamischen) Welt basierende Ideologie zurückzuführen ist und auf der Makroebene auf den Zerfall modernistischer Ideologien wie Sozialismus, Nationalismus oder Liberalismus sowie die starke Präsenz dschihadistischer Ideologien in der Öffentlichkeit.

Wie bedeutend auch immer die von Dschihadist*innen konstruierten kollektiven Identitäten für die Rekrutierung junger Menschen sind, dürfen die Individuen nicht als passive Rezipient*innen dieser Identitätsangebote konzipiert werden. Vielmehr prägen sie diese durch ihr dschihadistisches Engagement mit, sei dies durch ihre 'heroischen' Handlungen, virtuelle Kommunikation, ihre Mitwirkung in Propaganda usw. Mit anderen Worten handelt es sich hier um einen dialektischen Prozess, in welchem kollektive Identitäten durch ein Wechselspiel zwischen den Mikro-, Meso- und Makroeben kontinuierlich umgestaltet werden. Ein besseres Verständnis dieser Dialektik bedürfte vertiefter theoretischer Reflektion wie auch empirischer Auseinandersetzung. Zudem müssten wir besser verstehen, welche Rolle die Mesoebene als Bindeglied zwischen Mikro- und Makroebenen spielt und wie identitäre Konstruktionsleistungen auf dieser Ebene, etwa in dschihadistischen Milieus, Zellen, Netzwerken und Moscheen, funktionieren.

Forschungen, die Kenntnisse über die Genese sowie Merkmale dschihadistischer Identitäten vermitteln, haben unser Verständnis dieser Phänomene erweitert. Diese Studien bleiben aber disparat und fragmentiert. Künftige Studien sollten stärker in Richtung einer Konvergenz arbeiten, damit die gewonnen Erkenntnisse zur Akkumulation des Wissens und Vertiefung des Verständnisses dieses Sachverhalts beitragen können. Wie in diesem Artikel argumentiert, sollten Studien und ihre Befunde aber auch theoretische und methodologische Zugänge der Forschung über rechts- und linksradikalen Terrorismus sowie der Kultforschung integrie$\mathrm{ren}^{28}$. Des Weiteren bleibt es zu überprüfen, ob und inwiefern bei der Erforschung dieses $\mathrm{Zu}$ sammenhangs zwischen dschihadistisch engagierten Individuen im globalen Norden und denjenigen in den islamisch geprägten Kontexten zu unterscheiden ist.

Schliesslich darf ein identitätsbezogener Fokus nicht zur Vernachlässigung sozietaler Rahmenbedingungen führen. Vielmehr sollte dieser der Wechselwirkung zwischen Identität und Struktur Rechnung tragen. Wie wirken sich Entwicklungen wie technologischer Wandel,

\footnotetext{
${ }^{28}$ In ihren Recherchen über islamistische und rechtsradikale Gruppierungen in Belgien, Deutschland, Frankreich und Grossbritannien hat die Publizistin Ebner (2018) aufgezeigt, dass diese beiden Bewegungen sich nicht nur sehr ähnlich sind, sondern dass sie sich auch wechselseitig radikalisieren.
} 
staatliche Repressalien, Wandel der Öffentlichkeit im globalen Norden, politische Umwälzungen in islamisch geprägten Gesellschaften, Niederlagen der Dschihadist*innen bei den Kampfhandlungen und ähnliches mehr ${ }^{29}$ auf die individuellen wie auch kollektiven Identitäten der Dschihadist*innen aus? Und wie sieht die Wirkung der Identität auf die Struktur aus? Kann von einer Evolution dschihadistischer Ideologie gesprochen werden und wenn ja, wie lässt sich diese in Wechselwirkung mit strukturellen Faktoren erklären? Antworten auf diese und ähnliche Fragen würden unser Verständnis der Genese und Merkmale dschihadistischer Identitäten erheblich erweitern und vertiefen.

\section{Danksagung}

Der Autor bedankt sich bei den externen Gutachterinnen und Gutachtern und den Redaktorinnen und Redaktoren des Journals sozialpolitik.ch für die wertvollen Hinweise und Kommentare.

\section{Deklaration von Interessenkonflikten}

Der Autor deklariert keine Interessenkonflikte in Bezug auf Forschung, Autorinnenschaft und Publikation des Artikels.

\section{Finanzierung}

Der Autor hat keine finanzielle Unterstützung für die Forschung, die Autorinnenschaft und die Publikation dieses Artikels erhalten.

\section{Literaturverzeichnis}

Agnew, Robert (2010). A general strain theory of terrorism. Theoretical Criminology, 14(2), 131-153.

Al-Muhajirah, Umm Sumayyah (2015). The Twin Halves of the Muhajirin. Dabiq, March 1, 2015, 3237.

Anderson, Benedict R. (1983). Imagined communities: reflections on the origin and spread of nationalism. London: Verso.

Ashour, Omar (2015). Egypt's Revolution and the Transformation of Armed Islamist Movements towards Unarmed Activism. In: Dudouet, Véronique (Ed.). Civil Resistance and Conflict Transformation. Transitions from Armed to Nonviolent Struggle. London: Routledge, 173-189.

Atran, Scott (2010). Talking to the Enemy: Violent Extremism, Sacred Values, and What it Means to Be Human. London: Allen Lane.

\footnotetext{
${ }^{29}$ Für eine Diskussion der strukturellen Ursachen des Dschihadismus in Europa siehe etwa Schuurman et al. (2018).
} 
Behnam, Said T., und Hazim Fouad (Hrsg.) (2014). Salafismus: Auf der Suche nach dem wahren Islam. Freiburg im Breisgau: Herder.

Bjørgum, Maren H. (2016). Jihadi Brides: Why Do Western Muslim Girls Join ISIS?. Global Politics Review, 2(2), 91-102.

Bornschier, Volker (2007). Konflikt, Gewalt, Kriminalität und abweichendes Verhalten: Ursachen, Zeitund Gesellschaftsvergleiche. Zürich: LVB.

Brewer, Marilynn B. (2001). The many faces of social identity: Implications for political psychology. Political psychology, 22(1), 115-125.

Casutt, Géraldine (2018). Quand le djihadiste est une femme. Inflexions, 2(38), 87-96.

Cottee, Simon, and Keith Hayward (2011) Terrorist (E)motives: The Existential Attractions of Terrorism. Studies in Conflict \& Terrorism, 34(12), 963-986.

Ebner, Julia (2018). Wut. Was Islamisten und Rechtsextreme mit uns machen. Darmstadt: Theiss Verlag.

Eickelpasch, Rolf und Claudia Rademacher (2015). Identität. Bielfeld: transcript Verlag.

Eser Davolio, Miryam, Elisa Banfi, Milena Gehrig, Brigitta Gerber, Burim Luzha, Eva Mey, Ilona Möwe, Dominik Müller, Isabelle Steiner, Dilyara Suleymanova, Carole Villiger und Laurent Wicht (2015). Hintergründe jihadistischer Radikalisierung in der Schweiz: eine explorative Studie mit Empfehlungen für Prävention und Intervention. Zürich: ZHAW, https://projektdaten.zhaw.ch/Research/Projekt00002044/Schlussbericht Jihad.Radikalisierung.pdf (Zugriff am 12.02.2020)

Esposito, John L. (2002). Unholy war: terror in the name of Islam. Oxford: Oxford University Press.

Frindte, Wolfgang, Brahim B. Slama, Nico Dietrich, Daniela Pisoiu, Milena Uhlmann und Melanie Kausch (2016). Wege in die Gewalt: Motivationen und Karrieren salafistischer Jihadisten. (HSFKReport, 3/2016). Frankfurt am Main: Hessische Stiftung Friedens- und Konfliktforschung.

Gaub, Florence (2016). The cult of ISIS. Survival, 58(1), 113-130.

González Mendelejis, Nathan (2019). Toward a New Typology of Sunni Jihad. Studies in Conflict \& Terrorism, 1-22, DOI: 10.1080/1057610X.2018.1520797.

Gooren, Henri (2010). Religious conversion and disaffiliation: tracing patterns of change in faith practices. New York: Palgrave Macmillan.

Günther, Christoph, Mariella Ourghi, Susanne Schröter und Nina Wiedl (2016). Dschihadistische Rechtfertigungsnarrative und mögliche Gegennarrative, HSFK-Report Nr. 4/2016 (HSFK Reportreihe „Salafismus in Deutschland“, hrsg. von Janusz Biene, Christopher Daase, Svenja Gertheiss, Julian Junk, Harald Müller).

Haddad Kreidie, Lina, and Kristen Renwick Monroe (2002). Psychological Boundaries and Ethnic Conflict: How Identity Constrained Choice and Worked to Turn Ordinary People into Perpetrators of Ethnic Violence during the Lebanese Civil War. International Journal of Politics, Culture, and Society, 16(1), 5-36.

Hafez, Mohammad M. (2009). Jihad after Iraq: lessons from the Arab Afghans. Studies in Conflict \& Terrorism, 32(2), 73-94. 
Hafez, Mohammed M., and Creighton Mullins (2015). The radicalization puzzle: A theoretical synthesis of empirical approaches to homegrown extremism. Studies in Conflict \& Terrorism, 38(11), 958975.

Heck, Paul L. (2004). Jihad revisited. Journal of Religious Ethics, 32(1), 95-128.

Hegghammer, Thomas (2010). The rise of Muslim foreign fighters: Islam and the globalization of Jihad. International Security, 35(3), 53-94.

Hegghammer, Thomas (2011). Global jihadism after the Iraq War. In: Volpi, Frédéric (Ed.) Political Islam: a critical reader. New York: Routledge, 296-316.

Hegghammer, Thomas, and Stéphane Lacroix (2007). Rejectionist Islamism in Saudi Arabia: The Story of Juhayman al- 'Utaybi Revisited. International Journal of Middle East Studies, 39(1), 103-122.

Hehn, Jochen (1996). Jetzt fährt die Angst wieder mit: Trauer, Wut, Hilflosigkeit: Frankreich am Tag nach dem jüngsten Attentat. Die Welt, 05.12.1996.

Hemmingsen, Ann-Sophie (2010). The Attractions of Jihadism: An Identity Approach to Three Danish Terrorism Cases and the Gallery of Characters around Them. Copenhagen: University of Copenhagen.

Jouili, Jeanette S., and Schirin Amir-Moazami (2006). Knowledge, Empowerment and Religious Authority among pious Muslim Women in France and Germany. The Muslim World, 96(4), 617-642.

Kepel, Gilles (2006). Jihad: The trail of political Islam. London: IB Tauris.

Kepel, Gilles (2011). The origins and development of the Jihadist movement: from anti-communism to terrorism. In: Volpi, Frédéric (Ed.) Political Islam: a critical reader. New York: Routledge, 255-270.

Keupp, Heiner, Thomas Ahbe, Wolfgang Gmür, Renate Höfer, Beate Mitzscherlich, Wolfgang Kraus und Florian Straus (2008). Identitätskonstruktionen. Das Patchwork der Identitäten in der Postmoderne. Reinbek bei Hamburg: Rowohlt.

Khosrokhavar, Farhad (2009). Inside Jihadism. Understanding Jihadi Movements Worldwide. Boulder/London: Paradigm Publishers.

Kraus, Wolfgang, und Beate Mitzscherlich (1997): Abschied vom Großprojekt. Normative Grundlagen der empirischen Identitätsforschung in der Tradition von James E. Marcia und die Notwendigkeit ihrer Reformulierung. In: Keupp, Heiner, und Renate Höfer (Hrsg.). Identitätsarbeit heute: Klassische und aktuelle Perspektiven der Identitätsforschung. Frankfurt a.M.: Suhrkamp, 149-173.

Lindemann, Thomas (2018). Agency (mis) recognition in international violence: the case of French jihadism. Review of International Studies, 44(5), 922-943.

Lifton, Robert (1999). Destroying the World to Save It: Aum Shinrikyo, Apocalyptic Violence, and the New Global Terrorism. New York: Metropolitan Books, Henry Holt.

Lofland, John, and Rodney Stark (1965). Becoming a World-Saver: A Theory of Conversion to a Deviant perspective. American Sociological Review, 30(6), 862-875.

Lucius-Hoene, Gabriele und Arnulf Deppermann (2004). Rekonstruktion narrativer Identität. Ein Arbeitsbuch zur Analyse narrativer Interviews. Wiesbaden: VS Verlag.

Lueders, Michael (1997). Zwischen Bürgerkrieg und Blutrache: Die Massaker in Algerien nehmen kein Ende. Unternimmt die Regierung genug, den Terror zu unterbinden? Die Zeit, 5.09.1997. 
Maddox, Graham (2003). The "crusade" against evil: Bush's fundamentalism. Australian Journal of Politics \& History, 49(3), 398-411.

Marcia, James E. (1966). Development and validation of ego-identity status. Journal of Personality and Social Psychology, 3(5), 551-558.

Marlowe, Lara (2014). “Holocaust makes Israelis think international law doesn't apply”: Gideon Levy was the most outspoken critic of Israel's war in Gaza. The Irish Times, 11.09.2014, https://www.irishtimes.com/news/world/holocaust-makes-israelis-think-international-law-doesnt-apply-1.1924554 (Zugriff am 10.03.2020).

McGilloway, Angela, Priyo Ghosh, and Kamaldeep Bhui (2015): A systematic review of pathways to and processes associated with radicalization and extremism amongst Muslims in Western societies. International Review of Psychiatry, 27(1), 39-50.

Meeus, Wim (2015). Why do young people become Jihadists? A theoretical account on radical identity development. European Journal of Developmental Psychology, 12(3), 275-281.

Meiler, Oliver (1998). Gemetzel ohne Ende. Tagesanzeiger, 5.01.1998.

Moghadam, Assaf (2008a). The globalization of martyrdom: Al Qaeda, Salafi Jihad, and the diffusion of suicide attacks. Baltimore: The Johns Hopkins University Press.

Moghadam, Assaf (2008b). The Salafi-jihad as a religious ideology. CTC Sentinel, 1(3), 14-16.

Murawiec, Laurent (2008). The Mind of Jihad. Cambridge: Cambridge University Press.

Nedza, Justyna (2014): Salafismus - Überlegungen zur Schärfung einer Analysekategorie. In: Behnam, Said T., und Hazim Fouad (Hrsg.). Salafismus: Auf der Suche nach dem wahren Islam. Freiburg im Breisgau: Herder, 80-105.

Nesser, Petter (2010). Joining Jihadi Terrorist Cells in Europe: Exploring Motivational Aspects of Recruitment and Radicalization. In: Ranstorp, Magnus (Ed.). Understanding Violent Radicalisation: Terrorist and Jihadist Movements in Europe. New York: Routledge, 87-114.

Nollert, Michael (2019). Auf der Abkürzung ins Paradies: KonvertitInnen als Rekrutierungspotenzial dschihadistischer Gegenkultur. Sozialpolitik.ch, 2/2019 - FORUM.

Paloutzian, Raymond F. (2005). Religious Conversion and Spiritual Transformation. In: Paloutzian, Raymond F., and Crystal L. Park (Eds.). Handbook of the Psychology of Religion and Spirituality. New York: Guilford Press, 331-347.

Parashar, Swati (2010). The Sacred and the Sacrilegious: Exploring Women's 'Politics' and 'Agency' in Radical Religious Movements in South Asia. Totalitarian Movements and Political Religions, 11(34), 435-455.

Pilz, Gunter A. (1994). Jugend, Gewalt und Rechtsextremismus: Möglichkeiten und Notwendigkeiten politischen, polizeilichen, (sozial-)pädagogischen und individuellen Handelns. Münster, Hamburg: Lit Verlag.

Pisoiu, Daniela (2012). Islamist radicalisation in Europe: an occupational change process. London: Routledge.

Rahimullah, Riyad H., Stephen Larmar, and Mohamad Abdalla (2013). Understanding violent radicalization amongst Muslims: A review of the literature. Journal of Psychology and Behavioral Science, $1(1), 19-35$. 
Roald, Anne Sofie (2012). The conversion process in stages: new Muslims in the twenty-first century. Islam and Christian-Muslim Relations, 23(3), 347-362.

Robinson, Glenn E. (2017). The Four Waves of Global Jihad, 1979-2017. Middle East Policy, 24(3), 7088.

Rodríguez-Carballeira, Álvaro, Javier Martín-Peña, Carmen Almendros, Jordi Escartín, Clara Porrúa, and Massimo Bertacco (2010). A Psychosocial Analysis of the Terrorist Group as a Cult. International Journal of Cultic Studies, 1(1), 49-60, https://www.icsahome.com/articles/a-psychosocialanalysis-of-the-terrorist-group-rodriguez-ijcs-1-1 (Zugriff am 02.04.2020).

Roy, Oliver (2003). EuroIslam: The Jihad Within? The National Interest. March 1, 2003. https://nationalinterest.org/article/euroislam-the-jihad-within-517?page $=0 \% 2 \mathrm{C} 2 \quad$ (Zugriff am 21.05.2020).

Roy, Olivier (2007). Islamic Terrorist Radicalisation in Europe. In: Amghar, Samir, Amel Boubekeur, and Michael Emerson (Eds.) European Islam. Challenges for public policy and society. Brussels: Centre for European Policy Studies, 52-60.

Rushchenko, Julia (2017). Converts to Islam and Homegrown Jihadism. London: Centre on Radicalization and Terrorism at the Henry Jackson Society.

Schmidhuber, Martina (2010). Der Prozess personaler Identitätsbildung und die Rolle von Institutionen. Wien: Lit Verlag.

Schulze, Reinhard (2007). Islamistischer Terrorismus und die Hermeneutik des Terrors. In: WohlrabSahr, Monika, und Levent Tezcan (Hrsg.). Konfliktfeld Islam in Europa, Soziale Welt, Sonderband $17,77-110$.

Schuurman, Bart, Edwin Bakker, and Quirine Eijkman (2018). Structural influences on involvement in European homegrown jihadism: A case study. Terrorism and Political Violence, 30(1), 97-115.

Sedgwick, Mark (2015). Jihadism, narrow and wide: The dangers of loose use of an important term. Perspectives on Terrorism, 9(2), 34-41.

Sen, Amartya K. (2006). Identity and violence: The illusion of destiny. London: Allen Lane.

Sheikhzadegan, Amir (2003). Der Griff des politischen Islam zur Macht: Iran und Algerien im Vergleich. Dissertation. Bern [etc.]: Peter Lang Verlag.

Sheikhzadegan, Amir (2020). From Rigid to Moderate Salafism: Paths of (Re-)Conversion to Islam among Activists of a Muslim Organisation in Switzerland. Journal of Muslims in Europe, 9(2), Special Issue: Salafism in Europe. Empirical Approaches, edited by Sabine Damir-Geilsdorf and Mira Menzfeld, 196-219.

Sherif, Mohamed Ahmed (1975). Ghazali's Theory of Virtue. Albany: State University of New York Press.

Sjoberg, Laura (2018). Jihadi brides and female volunteers: Reading the Islamic State's war to see gender and agency in conflict dynamics. Conflict Management and Peace Science, 35(3), 296-311.

Slavicek, David J. (2008). Deconstructing the shariatic justification of suicide bombings. Studies in conflict \& terrorism, 31(6), 553-571.

Stahelski, Anthony (2005). Terrorists are made not born: Creating terrorists using social psychological conditioning. Cultic Studies Review, 4(1), 30-40. 
Stern, Jessica (2003). Terror in the Name of God: Why Religious Militants Kill. New York: HarperCollins.

Stern, Jessica, and John M. Berger (2015). ISIS: The State of Terror. New York: HarperCollins.

Straub, Jürgen (2000). Identitätstheorie, empirische Identitätsforschung und die „postmoderne“ armchair psychology. Zeitschrift für qualitative Bildungs-, Beratungs- und Sozialforschung, 1(1), 167194.

Straub, Jürgen (2018). Multiple Identitäten in modernen Gesellschaften. Zeitschrift für Politikwissenschaft, 28(2), 205-216.

Susman, Warren I. (1979). 'Personality' and the making of twentieth century culture. In: Higham, John, and Paul K. Conkin (Eds.). New directions in American intellectual history, Baltimore, MD: John Hopkins University Press, 212-226.

Tajfel, Henri, and John C. Turner (1986). The Social Identity Theory of Intergroup Behavior. In: Worchel, Stephen, and William G. Austin (Eds.). Psychology of Intergroup Relations. Chicago: Nelson Hall, 7-24.

Tirozzi, Gianluca (2019). The IS's symbols and values, from secularism to Jihad. Video analysis of Al Furat media foundation main contents. Phd Course in Applied Social Sciences, Working Papers Series, no. 3/2019. Rom: Sapienza University of Rome, Dipartimento di Scienze Sociali ed Economiche.

Travisano, Richard V. (1970). Alternation and conversion as qualitatively different transformations. In: Stone, Gregory P., and Harvey A. Farberman (Eds.). Social Psychology Through Symbolic Interaction. Waltham, MA.: Ginn-Blaisdell, 594-606.

Van den Berg, Dirk (2018). Urknall des Terrors? Dokumentarfilm. ARD-alpha, 75 Minuten.

Van Stekelenburg, Jacquelien (2013). Collective identity. In: Snow, David A., Donatella Della Porta, Pieter G. Klandermans, and Doug McAdam (Eds.). The Wiley-Blackwell encyclopedia of social and political movements. Malden: Wiley-Blackwell, 219-225.

Veelken, Ludger (1978). Identitätskrise und Terrorismus - Sozialpsychologische Aspekte personaler Wesensmerkmale des Terrorismus. In: Schwind, Hans-Dieter (Hgs.). Identitätskrise und Terrorismus. Ursachen des Terrorismus in der Bundesrepublik Deutschland. Berlin/New York: De Gruyter, 79-98.

Venema, Henry I. (2000). Identifying Selfhood: Imagination, Narrative, and Hermeneutics in the Thought of Paul Ricoeur. Albany: State University of New York Press.

Wagemakers, Joas (2017). Revisiting Wiktorowicz: Categorising and Defining the Branches of Salafism. In: Cavatorta, Francesco, and Fabio Merone (Eds.). Salafism After the Arab Awakening: Contending with People's Power. London: Hurst \& Company, 7-24.

Wiktorowicz, Quintan (2005). A Genealogy of Radical Islam. Studies in Conflict \& Terrorism, 28(2), 75-97.

Wiktorowicz, Quintan, and Karl Kaltenthaler (2006). The rationality of radical Islam. Political Science Quarterly, 121(2), 295-319. 